\title{
DECODING VIEWS AND SENTIMENTS OF PROGRAM HEADS TOWARDS THE SUPERVISION OF INSTRUCTION DURING THE COVID-19 PANDEMIC
}

\author{
Jesus M. Meneses III ${ }^{1}$, Karen W. Cantilang ${ }^{2}$, Delbert A. Dala ${ }^{3}$, \\ Jovito B. Madeja ${ }^{4}$ \\ ${ }^{1}$ Instructor, Department of Computer Science, Eastern Samar State University, Borongan Eastern \\ Samar, Philippines \\ ${ }^{2}$ Instructor, Department of Information Technology, Eastern Samar State University, Borongan \\ Eastern Samar, Philippines \\ ${ }^{3}$ Instructor, Department of Natural Sciences, Eastern Samar State University, Borongan Eastern \\ Samar, Philippines \\ ${ }^{4}$ Assistant Professor, Graduate School, Eastern Samar State University, Borongan Eastern Samar, \\ Philippines
}

Article DOI: $\underline{\text { https://doi.org/10.36713/epra8863 }}$ DOI No: 10.36713/epra8863

\begin{abstract}
The purpose of this study was to decode the hidden views and sentiments from the collated written responses of Eastern Samar State University's Program Heads regarding supervision of instructions amidst the COVID-19 pandemic. This study utilized Exploratory Sequential Mixed Method to explore and understand the perspective or sentiments of Eastern Samar State University program heads towards supervision of instruction in the midst of the COVID-19 pandemic. Data were collected/collated from the participants indirectly using an interview questionnaire containing an open-ended question. The same were processed and analyzed using an open-source machine learning software called Orange toolbox (Demsar et al., 2013) wherein pre-processing, sentiment analysis and topic modelling built-in tools were utilized. The results showed that the most prominent words generated by the machine learning tool from the text file of responses are the words pandemic, performance, program, learning, difficult, supervision, instruction, internet, faculty, online students, teaching, delivery confusing, challenging, poor and connectivity. The dominant sentiment associated thereof lean towards negative polarity which implicate negative sentiments. Hidden topics were automatically generated by the machine which allowed the researchers to come up with the following related themes: "Impact of pandemic in the supervision of instruction of faculty and learning of students", "Challenges in the delivery of instruction and supervision due to poor internet connectivity", and "Strategic role of online modalities and connectivity in supervision and delivery of instruction". There are limited researches navigating in text mining and sentiment analysis with the use of Orange toolbox particularly those that deals with supervision of instruction in a Philippine State University. There are related studies using machine learning software, but nothing like this study directed towards a specific gap in specific locale.
\end{abstract}

KEYWORDS: Pandemic, Latent Semantic Indexing, Orange Toolbox, Sentiment Analysis, Thematic Analysis. 


\section{INTRODUCTION}

The educational systems around the world have experienced disruptions in the normal conduct of classes and other related school activities because of the COVID-19 pandemic. Most learning institutions have shifted to online teaching which has caused disengagement of students from the instruction delivered by teachers (Mette, 2020). As a result, educational supervisors faces many challenges and opportunities to maximize learning for both teachers and students. The role of supervision in education during this time of pandemic is focused on providing support for educators as well as children and young people with issues affecting their well-being/mental health such as stress (Lofthouse, 2020). However, most of the school heads were caught between the plans made by the administration and the needs of teachers and students. As such, it has been necessary for them to utilize their skills and education to tackle new leadership challenges from creative perspectives. Fendi et al. 2021, emphasized the need for innovation in the implementation of supervision during the COVID-19 pandemic. Apart from enhancing teacher's capability in dealing with the challenges in the delivery of instruction due to prohibition of face to face interaction, mental health support was suggested as one of the priority areas. The Eastern Samar State University, being in a similar situation, has struggled to keep up during this time of health crisis. The administration, deans, program heads, and instructors are anxiously engaged in complying with its mandated tasks. Extensive efforts are continuously being exerted to maintain accessibility to quality instruction by all stakeholders. Policies and procedures are fluid and continuously updated in order to address the current needs of the situation. However, these efforts focused more on the enhancement of abilities particularly in dealing with technologies design to improve delivery of instruction, but failed to recognize the mental health aspect suggested by Fendi et al. (2021). Barsade \& O'Niel (2016) and Briner (1999) recognized emotion or sentiment as central in building a healthy work culture in an organization across roles and industries. Positive emotions are consistently associated with better performance at various organizational levels while (with certain exceptions), negative emotions such as group anger, sadness, fear, and the like usually lead to negative outcomes, including poor performance. Archana \& Kishore (2017) considers the same as a valuable part of human nature that have profound relevance in education sector particularly in its vital role in policy making. With very limited studies navigating in this area, the researcher deemed it necessary to conduct this study to explore and unfold the hidden overall sentiments behind the program head's written perceptions toward supervision of instruction amidst the COVID-19 pandemic.

\section{OBJECTIVES}

This study focused on exploring the perceptions/responses collated from Program Heads of Eastern Samar State University Main Campus regarding supervision of instructions during this pandemic. The purpose was to decode their hidden views and sentiments using a machine learning tool called Orange Toolbox (Demsar et al., 2013).

Specifically, this study aimed to achieve the following:

1. Identify the most prominent words from the text corpus.

2. Determine the overall sentiments of the program heads in the text corpus towards supervision of instructions during this COVID-19 pandemic.

3. Uncover hidden topics in the text corpus and develop relevant themes.

\section{METHODOLOGY \\ Research Design}

This study utilized an Exploratory Sequential Mixed Methods to explore and understand the supervisory perspective or sentiments of ESSU main program heads towards supervision of instruction in the midst of the COVID-19 pandemic. This method is an approach which combines qualitative and quantitative data collection and analysis in a sequence of phases (Creswell \& Plano Clark, 2018)

\section{Data Gathering}

- Participants/Respondents - Total enumeration of program heads in ESSU Borongan.

- Indirect interview was conducted using a questionnaire. In it is a single open-ended question "What can you say about the supervision of instruction during this Covid19 pandemic?" and a consent statement which ensures that responses thereof are of the participants free will or act. This was design to elicit unstructured information pertaining to the participant's/respondent's view towards supervision of instruction during this COVID-19 pandemic. 


\section{Data Analysis}

The initial phase started with data collection and pre-processing of qualitative information, then followed by quantitative data generation and analysis with a concluding phase of data interpretation utilizing the two results (Berman, 2017). To support the intention of the research, this study employed Sentiment analysis and Topic Modelling algorithm under the Orange free software application.

\section{Text Pre-processing and Cleaning}

Responses from the program heads were encoded using the Notepad application and saved as text files separately. All responses underwent text pre-processing and cleaning. In the pre-processing stage, filtering of keywords was performed and relationships within the text were identified in the process called pattern matching. The pre-processing and cleaning methods performed in this research were:

Stop words Removal Methods: A stop word may be identified as a word that has the same likelihood of occurring in those documents not relevant to a query as in those documents relevant to the query.

Stemming Method: This method is used to identify the root/stem of a word. For example, the words connect, connected, connecting, and connections, all can be stemmed to the word "connect". The idea of stemming is to reduce the total number of distinct terms in a document or a query which in turn will decrease the processing time of the final output. In this research, N-Gram Stemmer was utilized as it is language independent and hence very useful in many applications. An N-gram is an N-character slice of a longer string.

Term Frequency-Inverse Document Frequency: Term Frequency-Inverse Document Frequency (TFIDF) is a numerical statistic which shows how vital a word is to a document in a collection. The TF- IDF is often used as basis in information retrieval and text mining. The value of TF-IDF increases proportionally to the number of times a word appears in the text file but isn't affected by the frequency of the word in the corpus. This can help to control the fact that some words are generally more common than others. Term Frequency (TF) is specified as the number of times a term occurs in a document.

\section{Step 2. VADER Sentiment Analysis}

VADER (Valence Aware Dictionary for sEntiment Reasoning) is a sentiment analysis model used to determine the polarity (positive/negative/neutral) and intensity (strength) of the emotion of a corpus or set of documents. VADER, introduced in 2014, uses a human-centric approach that combines qualitative analysis and empirical validation by using human evaluators and crowdsourcing. To quantify the emotion of a word, VADER relies on a dictionary which maps words to emotion intensities called sentiment scores. The score of a text is computed by summing up the intensity value of each word in a corpus of documents. The scale used in measuring the emotion intensity ranges from -4 to +4 , where -4 is the largest negative score (most negative) and +4 as the most positive. Meanwhile, 0 (midpoint) is considered neutral. A normalization formula is applied to the overall emotion intensity to map the score within a range of -1 to +1 (Hutto and Gilbert, 2014) and (Calderon, 2017).

\section{Step 3. Topic Modelling (Latent Semantic Indexing)}

Determining the underlying sentiments in the collated data requires more than establishing its polarity - whether the same is leaning towards positivity, negativity or neutrality. It is equally important to uncover latent themes or topics in the text corpus by examining the pattern of words found therein. This can be done using unsupervised machine learning algorithm called Topic Modelling. Such a modelling technique is widely used in data mining for thematic analysis of text corpus. It finds human-readable structures in unstructured textual data, and one of the most widely used techniques is the Latent Semantic Indexing model.

Howes (2020) describes LSI as a process in Natural Language Processing (NLP) and a subset of linguistics and information engineering which focuses on how machines interpret human language. One important component of which is the distributional semantics wherein words with similar contextual meanings within large data sets are classified.

To make information retrieval accurate, LSI uses a mathematical method called Singular Value Decomposition (SVD). This method identifies the hidden contextual relationships between words.

SVD converts strings of words to its integral parts in a matrix to make calculations thereof simple and efficient. To isolate the words which comprise the main 'content' of a phrase, stop words such as conjunctions, pronouns and common verbs are removed using Stop Word Removal Method

Here's a quick example of how this might look: 


\section{The quick brown fox jumps over the lazy dog}

These processed words are then placed in a two dimensional (2D) table called Term Document Matrix (TDM). Such matrix shows the frequency with which each specific word (or term) occurs in the documents within a data set. A value of 1 is given when a work occurs in a document and 0 if none.

When words occur with the same general frequency in documents, it is called co-occurrence. Below is a basic example of a TDM, and how it assesses co-occurrence across multiple phrases:

Table 1. Sample TDM

\begin{tabular}{|l|c|c|c|c|c|c|c|}
\hline & Quick & Brown & Fox & Jumps & Over & Lazy & Dog \\
\hline $\begin{array}{l}\text { The quick brown } \\
\text { fox jumps over } \\
\text { the lazy dog }\end{array}$ & 1 & 1 & 1 & 1 & 1 & 1 & 1 \\
\hline $\begin{array}{l}\text { If the fox is } \\
\text { quick he can } \\
\text { jump over the } \\
\text { dog. }\end{array}$ & 1 & 0 & 1 & 0 & 1 & 0 & 1 \\
\hline $\begin{array}{l}\text { Foxes are quick. } \\
\text { Dogs are lazy. }\end{array}$ & 0 & 1 & 1 & 0 & 0 & 1 & 1 \\
\hline $\begin{array}{l}\text { Can a fox jump } \\
\text { over a dog? }\end{array}$ & 0 & 0 & 1 & 1 & 1 & 0 & 1 \\
\hline
\end{tabular}

SVD allows us to determine the patterns in word usage across all documents. LSI can use the relationships between words to better understand their sense, or meaning, in a specific context.

The above procedural narration can be summarized in Fig.1 showing the major activities taken to satisfy the contemplated end.

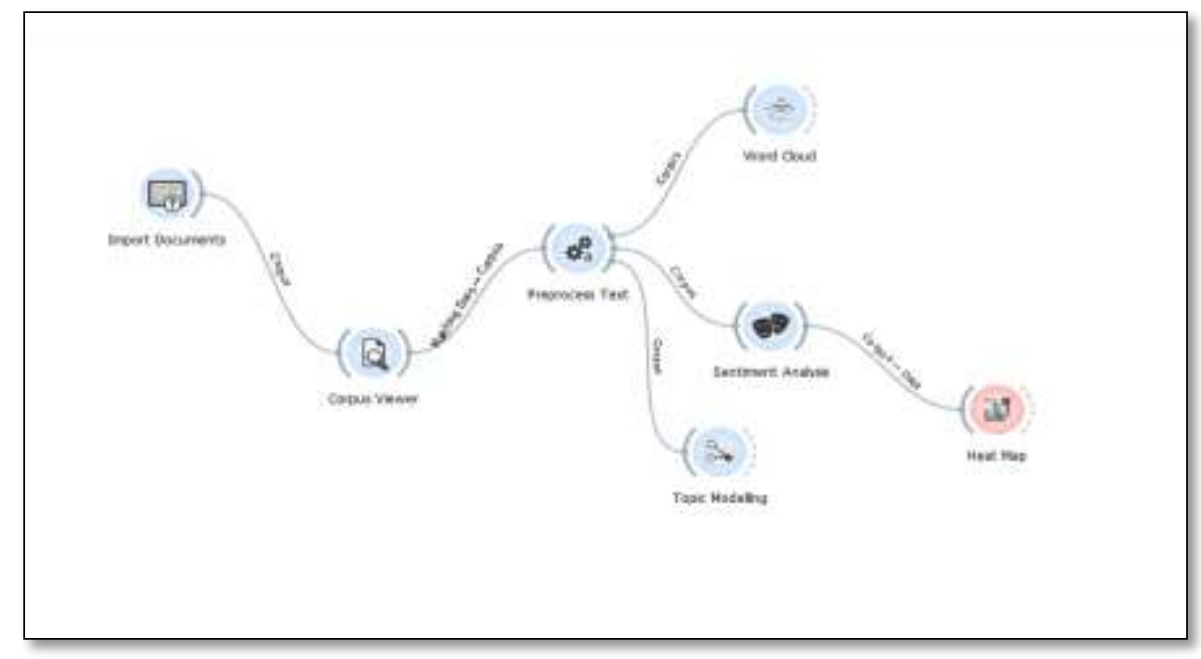

\section{Ethical Consideration}

The conduct of the study has complied with the principles of ethics in research such as the use of informed consent embedded in the questionnaire as well as confidentiality of the data. Risks and benefits in the conduct of the study were properly addressed.

\section{RESULTS AND DISCUSSION}

As shown in figure 2, the most prominent words generated by the machine learning tool from the text file of responses are pandemic, program, difficult, learning, supervision, instruction, internet, faculty, online, students, delivery, poor and connectivity. The philosophical assumption of these words navigates on the idea that the participants associate supervision, instruction, learning, program, faculty, with words like poor, and difficult and 
further associated with pandemic, online and connectivity among others. Such association presupposes the common view program heads insofar as the subject of the research inquiry is concern.

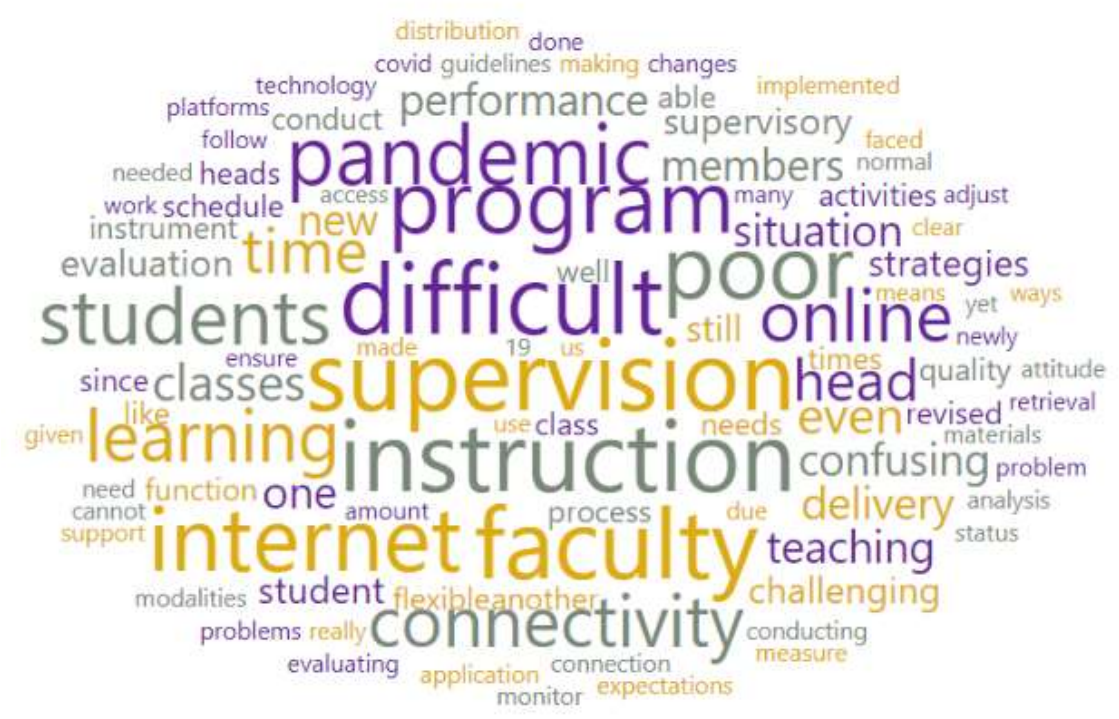

Fig. 2: Word Cloud generated from Orange toolbox's pre-processed text file

To determine the sentiment scores of the text corpus, the same was fed into a sentiment analysis algorithm. Table 2 shows the quantitative description of the underlying sentiments in the text corpus after subjecting the same with VADER method of sentiment analysis. What can be clearly deduced from the said matrix, based on the score under the compound column, is that, $\mathrm{Ph} 6, \mathrm{Ph} 8, \mathrm{Ph} 9, \mathrm{Ph} 12$, and $\mathrm{Ph} 15$ lean toward the positive sentiment polarity while $\mathrm{Ph} 1, \mathrm{Ph} 2, \mathrm{Ph} 3, \mathrm{Ph} 4, \mathrm{Ph} 5, \mathrm{Ph} 7, \mathrm{Ph} 10, \mathrm{Ph} 11, \mathrm{Ph} 13$ and $\mathrm{Ph} 14$, to the negative sentiment polarity.

Looking at the significant number of negative scores as compared to the positive ones, the participant's views or perceptions generally implicated negative sentiments towards supervision of instruction during this time of pandemic. Such implication was also consistent with the result shown in fig. 2, wherein the prominent words indicated association with negative words such as "poor" and "difficult". Such results were further affirmed with the use of the visualization tool Heat Map (Fig.3). Heat Map uses a color coding scheme to characterize the polarity of sentiments scores. Blue represents negativity and Yellow represents positivity. Any score that falls on a color other than blue or yellow are deemed neutral.

\begin{tabular}{|c|c|c|c|c|c|}
\hline title & $\begin{array}{l}\text { name } \\
\text { True }\end{array}$ & pos & neg & neu & compound \\
\hline 1 & ph1 & 0.064 & 0.342 & 0.594 & -0.9403 \\
\hline 2 & ph10 & 0.062 & 0.102 & 0.836 & -0.8781 \\
\hline 3 & ph11 & 0.046 & 0.084 & 0.87 & -0.3612 \\
\hline 4 & ph12 & 0.096 & 0.023 & 0.881 & 0.8862 \\
\hline 5 & ph13 & 0 & 0.23 & 0.77 & -0.9507 \\
\hline 6 & ph14 & 0.075 & 0.146 & 0.779 & -0.624 \\
\hline 7 & ph15 & 0.159 & 0 & 0.841 & 0.7506 \\
\hline 8 & ph2 & 0.071 & 0.138 & 0.791 & -0.34 \\
\hline 9 & ph3 & 0 & 0.136 & 0.864 & -0.7003 \\
\hline 10 & ph4 & 0.075 & 0.173 & 0.752 & -0.8313 \\
\hline 11 & ph5 & 0.083 & 0.14 & 0.777 & -0.8061 \\
\hline 12 & ph6 & 0.104 & 0 & 0.896 & 0.9529 \\
\hline 13 & ph7 & 0.085 & 0.213 & 0.702 & -0.9611 \\
\hline 14 & ph8 & 0.123 & 0.024 & 0.853 & 0.8402 \\
\hline 15 & ph9 & 0.042 & 0 & 0.958 & 0.3612 \\
\hline
\end{tabular}

Table 2: Sentiment Scores of Program Head (Ph)
Fig. 3: Heat Map

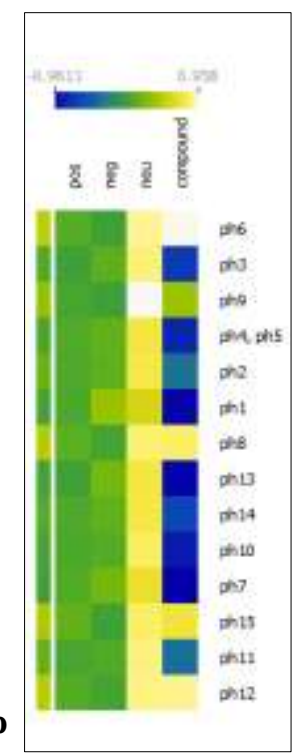


Table 3 shows the topic model as well as the corresponding themes generated after subjecting the text corpus to a topic modelling algorithm particularly Latent Semantic Indexing model. Three topics were autogenerated with 10 keywords (Categories) per topic. Based on the philosophical assumption of the researchers (Aryal, A., Gallivan, M., \& Tao, Y. Y., 2015), the first topic implicates the negative impact of pandemic in the supervision of instruction of faculty and the learning of students. As educators the researchers are quite aware of the challenges brought about by the pandemic the veracity of which is abundantly supported by varied scientific works such as in the work of Mette I. (2020) wherein meaningful, engaging pedagogical practices that meet the needs of student should be provided both by supervisors and educators. One common concern which often manifests in pandemic related studies is the issue of internet connectivity such as in the work of Dridi, M. et.al (2020) finding difficulty in the delivery of quality instruction and supervision under the implementation of a massive open online course (MOOC) due to poor internet connectivity, confirmed by topic 2 (see Table 3). With the continuing imposition by governments of pandemic induced lock downs, travel restrictions and mobility, instructors and students among others were left without much choice but to actively engage in remote transaction including academics with the use of ICT or online platforms. The use of such platforms undeniably became a crucial component of resiliency for humanity. Hence, Topic 3 (see Table 3), was detected, recognizing the strategic role of online modalities and connectivity particularly in supervision and delivery of instruction (Mahmood, S., 2021).

Table 3. Topic modelling and Thematic Analysis

\begin{tabular}{|c|l|l|}
\hline Topic & \multicolumn{1}{|c|}{ Topic Key Words } & \multicolumn{1}{|c|}{ Theme } \\
\hline 1 & $\begin{array}{l}\text { Supervision, poor, difficult, instruction, time, } \\
\text { students, learning, faculty, pandemic, program }\end{array}$ & $\begin{array}{l}\text { Impact of pandemic in the supervision of } \\
\text { instruction of faculty and learning of } \\
\text { students. }\end{array}$ \\
\hline $\mathbf{2}$ & $\begin{array}{l}\text { Difficult, connectivity, internet, online, confusing, } \\
\text { deliver, poor, challenging, supervision, since }\end{array}$ & $\begin{array}{l}\text { Challenges in the delivery of instruction and } \\
\text { supervision due to poor internet connectivity. }\end{array}$ \\
\hline $\mathbf{3}$ & $\begin{array}{l}\text { Modalities, connectivity, online, instruction, } \\
\text { delivery, even, able, strategies, time, supervisory }\end{array}$ & $\begin{array}{l}\text { Strategic role of online modalities and } \\
\text { connectivity in supervision and delivery of } \\
\text { instruction. }\end{array}$ \\
\hline
\end{tabular}

\section{CONCLUSION}

The purpose of this study was primarily to determine the overall sentiments of programs heads in Eastern Samar State University towards supervision of instruction amidst covid19 pandemic. The researcher believed that it was imperative for the university to consider the emotion aspect equally important with any other aspect that requires great deal of attention during this health crisis for the same is associated with faculty performance (Rapisarda, 2002). Participants were subjected to indirect interview wherein their perception towards the subject of inquiry were collected and processed using Orange toolbox. Built-in algorithms such as text mining tools, sentiment analysis and topic modelling were utilized to generate the pre-processed data for analysis.

The results showed that the most prominent words generated by the machine learning tool from the text file of responses as shown in Fig. 2, are the words pandemic, performance, program, learning, difficult, supervision, instruction, internet, faculty, online students, teaching, delivery confusing, challenging, poor and connectivity. The dominant sentiment associated thereof as shown in Table 1 and Fig.3, lean towards negative polarity which implicate negative overall sentiments by the participants. To explore further what these sentiments were all about, topic models were automatically generated by the machine allowing the researchers to come up with the following related themes: "Impact of pandemic in the supervision of instruction of faculty and learning of students", "Challenges in the delivery of instruction and supervision due to poor internet connectivity", and "Strategic role of online modalities and connectivity in supervision and delivery of instruction". However, due to the limited number of participants considered, the same lacks the character for generalization.

\section{RECOMMENDATION}

Sentiment Analysis or Opinion Mining plays a promising role in education policy making. Sentiments being a valuable part of human nature have profound relevance in every domain and education sector is no exception (Archana R \& Kishore B, 2017). With the results of the sentiment analysis showing a negative polarity insofar as the perspective of the program heads are concern towards their supervisory functions during this time of pandemic, the same invokes further investigation either for policy review or management purposes to mitigate if not 
eradicate such negative sentiments which may, have indirect or direct implication of the participants' performance. The underlying themes uncovered also suggest possible area of interest for further studies.

\section{REFERENCES}

1. Mahmood, S. (2021). Instructional strategies for online teaching in COVID-19 pandemic. Human Behavior and Emerging Technologies, 3(1), 199-203. https://onlinelibrary.wiley.com/doi/10.1002/hbe2.218

2. Fendi, Hasri; Hanafi, Imam; Monia, Fennt Ayu; Taufig, M. Aries; Putri, Rahmi Eka., (2021). Online-based academic supervision during the Covid-19 pandemic. Retrieved $\quad$ from https:// iopscience. iop.org/article/10 .1088/1742$6596 / 1779 / 1 / 012027 / p d f$

3. Lofthouse, Rachel. 2020. Does supervision help school leaders during the pandemic? A new research question. Retrieved from https://www.leedsbeckett.ac.uk/blogs/carnegie-education/2020/05/does super vision help/

4. Howes C. A. (2020). What is Latent Semantic Indexing. Retrieved from: https://www.oncrawl.com/technical-seo/whatis-latent-semantic-indexing/

5. Mette, I. (2020). Reflections on Supervision in the Time of COVID-19. Journal of Educational Supervision, 3 (3). https://doi.org/10.31045/jes.3.3.1

6. Dridi, M. A., Radhakrishnan, D., Moser-Mercer, B., \& DeBoer, J. (2020). Challenges of Blended Learning in Refugee Camps: When Internet Connectivity Fails, Human Connection Succeeds. The International Review of Research in Open and Distributed Learning, 21(3), 250-263. http://www.irrodl.org/index.php/irrodl/article/view/4770

7. Creswell, J. W., \& Plano Clark, V. L. (2018). Designing and conducting mixed methods research. Thousand Oaks, CA: SAGE. https://us.sagepub.com/en-us/nam/designing-and-conducting-mixed-methods-research/book241842

8. Berman EA. An Exploratory Sequential Mixed Methods Approach to Understanding Researchers' Data Management Practices at UVM: Integrated Findings to Develop Research Data Services. Journal of eScience Librarianship 2017; 6(1):e1104.https://doi.org/10.7191/jeslib.2017.1104.Retrievedfromhttps://escholarship.umassmed.edu/jeslib/vol6/iss1/7

9. Archana R.P N \& Kishore B. (2017). Role of Sentiment Analysis in Education Sector in the Era of Big Data: A Survey. International Journal of Latest Trends in Engineering and Technology Special Issue SACAIM 2017, pp. 022-024 eISSN:2278-621X

10. Calderon, P. (2017, April 10). http://datameetsmedia.com/vader-sentiment-analysis-explained/. Retrieved from http://datameetsmedia.com.

11. Barsade S. \& O'Neill O. (2016).Manage your emotional culture. Retrieved from: https://hbr.org/2016/01/manageyour-emotional-culture

12. Aryal, A., Gallivan, M., \& Tao, Y. Y. (2015). Using latent semantic analysis to identify themes in IS healthcare research.. https://citeseerx.ist.psu.edu/viewdoc/ download?doi=10.1.1.834.4857\&rep=rep1 \&type $=$ pdf

13. Hutto, C., \& Gilbert, E. (2014). VADER: A Parsimonious Rule-based Model for Sentiment Analysis of Social Media Text. Association for the Advancement of Artificial Intelligence. https://www.researchgate.net/ publication/ 275828927_VADER_A_Parsimonious_Rule-based Model for Sentiment Analysis of Social Media Text

14. Demsar J, Curk T, Erjavec A, Gorup C, Hocevar T, Milutinovic M, Mozina M, Polajnar M, Toplak M, Staric A, Stajdohar M, Umek L, Zagar L, Zbontar J, Zitnik M, Zupan B (2013) Orange: Data Mining Toolbox in Python, Journal of Machine Learning Research 14(Aug): 2349-2353. https://jmlr.org/ papers/volume14/ demsar13a/ demsar13a.pdf

15. Rapisarda, B. A. (2002). The impact of emotional intelligence on work team cohesiveness and performance. The International Journal of Organizational Analysis.

16. Briner R. B. (1999) The Neglect and Importance of Emotion at Work, European Journal of Work and Organizational Psychology, 8:3, 323-346, DOI: 10.1080/ 135943299398212 